\title{
Propuesta didáctica crítica para promover el comportamiento lector de textos multimodales digitales en la básica primaria ${ }^{1}$
}

Critical didactic proposal to promote the reading behavior of digital multimodal texts in primary school

\begin{abstract}
Gerzon Yair Calle-Álvarez²
gerzon.calle@udea.edu.co

Malca Irina Gómez-Sierra ${ }^{3}$

malca.gomez@udea.edu.co
\end{abstract}

https://doi.org/10.22209/rhs.v6n2a03

Recibido: septiembre 12 de 2018.

Aceptado: noviembre 23 de 2018.

\section{Resumen}

La didáctica crítica permite abordar temáticas sociales que emergen de los intereses y necesidades de los estudiantes. Para su acercamiento, se puede hacer uso de textos multimodales digitales, como posibilidad de acceso a la información y construcción de conocimientos. Este artículo es el resultado de una investigación que tuvo como objetivo valorar una propuesta didáctica crítica apoyada en textos multimodales digitales

1 Artículo original derivado del proyecto de investigación «El comportamiento lector en textos multimodales digitales en la básica primaria». En la Institución Educativa Luis Eduardo Díaz, Yondó, Antioquia, Colombia, durante el periodo julio 2016 - junio 2017

2 Integrante del grupo de investigación: Didáctica y Nuevas Tecnologías de la Universidad de Antioquia. Profesor de la Corporación Universitaria Remington.

3 Universidad de Antioquia. con estudiantes de tercer grado de básica primaria. El enfoque asumido fue cualitativo, bajo el método investigación-acción, y los instrumentos de recolección de información fueron un grupo de discusión, una guía de observación y un cuestionario a veinte estudiantes de grado tercero de una institución educativa pública, en el municipio de Yondó, Antioquia. En los resultados se destacan la relación de los textos multimodales digitales con el entorno social, la interacción con las Tecnologías de la Información y la Comunicación, y el trabajo en equipo, en el comportamiento lector de textos multimodales digitales, en estudiantes de básica primaria. En las conclusiones se plantea que la lectura resulta ser una práctica social para los estudiantes, en tanto aborde temáticas de su interés, que les permita intervenir, aportar ideas y experiencias de su cotidianidad, interactuar con otros sujetos desde diferentes espacios, de tal manera que el entorno en el que viven resulte ser un factor fundamental que favorezca su comportamiento lector.

Palabras claves: Básica primaria; Comportamiento lector; Didáctica crítica; Secuencia didáctica; Textos multimodales; Tecnologías de la Información y la comunicación (TIC). 


\section{Abstract}

Critical didactics allows addressing social issues that emerge from students' interests and needs. To implement this proposal, digital multimodal texts can be used, as a way to access information and build knowledge. This paper is the result of a research study that aimed to assess a critical didactic proposal built on digital multimodal texts and implemented with third grade elementary students. A qualitative approach was used under an action research methodology. The information was collected from twenty third grade students of a public educational institution in the municipality of Yondó (Antioquia) through three tools, namely, a discussion group, an observation guide and a questionnaire. The results section highlights the relationship between digital multimodal texts and the social environment, the interaction with information and communication technologies, and teamwork concerning the reading behavior of digital multimodal texts among elementary students. The paper concludes that reading is a social practice for students, as long as it addresses topics of interest that allow them to participate, contribute ideas and experiences of their daily life and interact with other individual from different settings, in such a way that the environment where they live becomes a critical factor favoring their reading behavior.

Keywords: Primary school; reading behavior; critical didactics; didactic sequence; multimodal texts; information and communication technologies (ICT).

\section{Introducción}

I hablar de didáctica crítica es necesario
retomar sus inicios en la teoría crítica de la escuela de Fráncfort quienes inmersos en una problemática social instan a la sociedad a defender un interés principal para ellos, como fue la emancipación. De tal manera que sus principios están orientados en la autonomía, la autodeterminación y la solidaridad que guían el concepto de educación. Uno de sus representantes es Klafki (1990), quien define la didáctica como: «el complejo total de las decisiones, presuposiciones, fundamentaciones y procesos de la decisión sobre todos los aspectos de la enseñanza» (p. 86). En tal sentido, aborda aspectos fundamentales en la educación como los objetivos, los contenidos, las formas de organización y realización, y medios de enseñanza y aprendizaje (Klafki, 1990), donde la teoría y la práctica convergen hacia un mismo objetivo, resultando difícil su separación.

Para Rodríguez (1997) la didáctica crítica es «la ciencia teórico-práctica que orienta la acción formativa, en un contexto de enseñanza-aprendizaje, mediante procesos tendencialmente simétricos de comunicación social, desde el horizonte de una racionalidad emancipadora» (p. 140). En este sentido, el profesor abandona su carácter dogmático y se involucra en los procesos de aprendizaje, reconstruyendo sus prácticas pedagógicas que favorezcan en los estudiantes la toma de decisiones, la interpretación, sus posturas frente a situaciones que emergen de su contexto social, la autorreflexión y la aplicación de valores que fortalecen su personalidad. 
Cuesta, Mainer, Mateos, Merchán y Vicente (2005) exponen la necesidad de concebir la didáctica crítica como una disciplina que más que conceptos, emerjan espacios de encuentro, de experiencias, cuestionamientos e interpretaciones, donde el sujeto construye su saber y donde el profesor no tiene un carácter dogmático. Los autores indican que «en la didáctica crítica es el docente que piensa, en cierto modo, contra sí mismo y contra su misma práctica, contra su pensamiento y contra su posición en el juego de las relaciones de poder dentro y fuera del aula» (p. 32). Por consiguiente, las prácticas pedagógicas se convierten en escenarios apropiados para estructurar las actividades que en la cotidianidad se orientan a los estudiantes, dando sentido a aquellos saberes que nacen de la necesidad de conocer y explorar como seres sociales que están inmersos en las diversas situaciones de su entorno, que inciden en la búsqueda de respuestas, a través del cuestionamiento, de la duda o la sospecha.

Al abordar la lectura de textos multimodales digitales surge la necesidad de dar una mirada hacia la implementación de una didáctica que relacione al sujeto con su realidad social y le permita tener mayor autonomía, conciencia de sus decisiones y racionalidad como la didáctica crítico-constructiva planteada por Klafki. Gimeno (1995) afirma que esta «se apoya en un concepto de educación que pretende posibilitar que todos los hombres desarrollen su capacidad de autodeterminación, de manera que puedan superar las condiciones sociales irracionales de dominio existentes en la sociedad» (p. 652), invita al sujeto a leer su contexto, a ser razonable frente a su realidad, más humano, a estar presto al diálogo y al respeto por el otro.

En la actualidad no se piensa en una didáctica rigurosa, impositiva. Por el contrario «la didáctica pretende generar alternativas consistentes para orientar las prácticas de enseñanza, hecho que le otorga un carácter propositivo» (Pérez Abril y Rincón Bonilla, 2009, p. 2). Estas propuestas parten del contexto social del sujeto, sus intereses y necesidades. Por lo anterior, se plantea una selección de contenidos orientados por objetivos pedagógicos que estén en función del aprendizaje del sujeto, pero encaminados a una educación crítico-social, que conlleven a la búsqueda de soluciones o reflexiones que se susciten. Todo ello alrededor de la implementación de medios de enseñanza ajustados a los objetivos que se desean alcanzar (Gimeno, 1995).

Igualmente, con el auge de las Tecnologías de la Información y la Comunicación (TIC), se ha evidenciado un aumento notable en su uso, por cuanto las lecturas en los soportes digitales se han fortalecido, por su fácil acceso, rapidez en la búsqueda de información e interacción con dichas herramientas. Gértrudix Barrio y Ballesteros Ávila (2014) afırman que «la incorporación de las TIC como recurso educativo en el aula de Educación Infantil ha fortalecido y mejorado el aprendizaje de los alumnos estudiados» (p. 9). De tal manera que fortalecen sus habilidades digitales y comunicativas.

Por lo anterior, se hace necesaria la implementación de propuestas didácticas que involucren las TIC como una herramienta que permita crear entornos que integren los sistemas semióticos y generen interacción y comunicación entre los participantes, por lo cual se hagan visibles los intereses de los estudiantes frente al proceso lector (Fernández-Díaz y Calvo, 2013). Por ello, cobra importancia el replantear las formas de aproximar a los estudiantes a la lectura desde una didáctica crítica. Partiendo de lo anterior, el presente artículo tiene como propósito mostrar los resultados de una investigación 
que tenía como objetivo valorar la propuesta didáctica crítica apoyada en textos multimodales digitales a través del desarrollo del comportamiento lector evidenciado en los estudiantes de tercer grado.

\section{Metodología}

Se determinó implementar la investigación-acción como método que permitió sistematizar la praxis educativa, así como establecer los momentos o fases estratégicas de planificación, ejecución, observación, reflexión y evaluación. Doria y Castro (2012) afırman que «la investigación-acción genera muchas posibilidades de intercambio, de diálogo, de colaboración, de comprensión, de empoderamiento y de transformación de los maestros, de manera situada; es decir, en terreno y con sentido autogestionario» (p. 2). De modo que sea posible autorreflexionar y realizar ajustes donde de manera cíclica se haga evidente y significativo el objetivo de la propuesta planteada.
El alcance de la presente investigación fue de carácter descriptivo. Hernández, Fernández y Baptista (2010) plantean que «los estudios descriptivos buscan especificar las propiedades, las características y los perfiles importantes de personas, grupos, comunidades o cualquier otro fenómeno que se someta a un análisis» (p. 80).

La investigación se desarrolló con 31 estudiantes, 13 niñas y 18 niños de tercer grado de básica primaria, de la Institución Educativa Luis Eduardo Díaz, zona urbana central del municipio de Yondó, Antioquia, pertenecientes a los estratos socioeconómicos 0 y 1 , con edades que oscilan entre los 7 y 12 años. Dicha investigación se implementó durante las clases de Lengua castellana, Ciencias Sociales, Ética y Valores y Tecnología e Informática. Las sesiones de trabajo se desarrollaron en el aula de clases y una sala de informática, con acceso a internet. A continuación, se presenta la Tabla 1, que explica las relaciones de las áreas mencionadas y las sesiones de trabajo de la secuencia didáctica:

Tabla 1. Áreas de trabajo durante las sesiones

\begin{tabular}{l|l|l}
\multicolumn{1}{c|}{ Sesión } & \multicolumn{1}{|c}{ Situaciónde lectura } & \multicolumn{1}{c}{ Áreas de trabajo } \\
\hline $\begin{array}{l}\text { Ciclo 1: } \\
\text { sesión 1 }\end{array}$ & Acuerdos de convivencia. & $\begin{array}{l}\text { Lengua Castellana, Ética y Valores, } \\
\text { Tecnología e Informática. }\end{array}$ \\
\hline $\begin{array}{l}\text { Ciclo 1: } \\
\text { sesión 2 } \\
\text { y 3 }\end{array}$ & $\begin{array}{l}\text { Lectura de textos narrativos } \\
\text { digitales. } \\
\text { Textos multimodales digitales de } \\
\text { los estudiantes. }\end{array}$ & $\begin{array}{l}\text { Lengua Castellana, Ética y Valores, } \\
\text { Tecnología e Informática. }\end{array}$ \\
\hline & $\begin{array}{l}\text { Lectura de textos expositivos } \\
\text { digitales. } \\
\text { Lectura de trabajos en Paint } \\
\text { elaborados por los estudiantes. } \\
\text { Lectura de textos propuestos por } \\
\text { sesión 1 }\end{array}$ & $\begin{array}{l}\text { Lengua Castellana, Ética y Valores, } \\
\text { lostudiantes. } \\
\text { Producción y lectura de textos } \\
\text { multimodales digitales elaborados } \\
\text { por los estudiantes: mapas } \\
\text { conceptuales, afiches digitales. }\end{array}$ \\
\hline
\end{tabular}

Tabla continua en la siguiente página. 


\begin{tabular}{|c|c|c|}
\hline Sesión & Situaciónde lectura & Áreas de trabajo \\
\hline $\begin{array}{l}\text { Ciclo 2: } \\
\text { sesión } 2\end{array}$ & $\begin{array}{l}\text { Lectura de textos multimodales } \\
\text { digitales propuestos por los } \\
\text { estudiantes. } \\
\text { Producción y lectura de afiches } \\
\text { digitales. }\end{array}$ & $\begin{array}{l}\text { Lengua Castellana, Ética y Valores, } \\
\text { Tecnología e Informática, Ciencias } \\
\text { Sociales. }\end{array}$ \\
\hline $\begin{array}{l}\text { Ciclo 2: } \\
\text { sesión } 3\end{array}$ & $\begin{array}{l}\text { Lectura de textos propuestos por } \\
\text { los estudiantes. } \\
\text { Producción y lectura de afiches y } \\
\text { cómics digitales. }\end{array}$ & $\begin{array}{l}\text { Lengua Castellana, Ética y Valores, } \\
\text { Tecnología e Informática, Ciencias } \\
\text { Sociales. }\end{array}$ \\
\hline $\begin{array}{l}\text { Ciclo 3: } \\
\text { sesión } 1\end{array}$ & $\begin{array}{l}\text { Lectura de textos argumentativos } \\
\text { digitales establecidos y } \\
\text { propuestos por los estudiantes. } \\
\text { Elaboración y lectura de afiches } \\
\text { digitales: acuerdos y desacuerdos. }\end{array}$ & $\begin{array}{l}\text { Lengua Castellana, Ética y Valores, } \\
\text { Tecnología e Informática, Ciencias } \\
\text { Sociales. }\end{array}$ \\
\hline $\begin{array}{l}\text { Ciclo 3: } \\
\text { sesión } 2\end{array}$ & $\begin{array}{l}\text { Lectura de textos argumentativos } \\
\text { digitales establecidos y } \\
\text { propuestos por los estudiantes. } \\
\text { Producción y lectura de texto } \\
\text { multimodal digital. }\end{array}$ & $\begin{array}{l}\text { Lengua Castellana, Ética y Valores, } \\
\text { Tecnología e Informática, Ciencias } \\
\text { Sociales. }\end{array}$ \\
\hline $\begin{array}{l}\text { Ciclo 3: } \\
\text { sesión } 3\end{array}$ & $\begin{array}{l}\text { Lectura de textos argumentativos } \\
\text { digitales establecidos y } \\
\text { propuestos por los estudiantes. } \\
\text { Lectura de argumentos } \\
\text { propuestos por los estudiantes. }\end{array}$ & $\begin{array}{l}\text { Lengua Castellana, Ética y Valores, } \\
\text { Tecnología e Informática, Ciencias } \\
\text { Sociales. }\end{array}$ \\
\hline
\end{tabular}

Fuente: elaboración propia.

Para el diseño de la propuesta didáctica crítica fue necesario revisar el currículo de la institución educativa y allí se pudo encontrar que su estructura, si bien trata de encajar bajo las normativas legales, requiere de una revisión más detallada, donde se visibilice el contexto social de los estudiantes que la conforman. Se hizo un análisis de la población investigada y se identificaron los intereses, necesidades y prioridades en su entorno social. En la actualidad, uno de sus mayores intereses es el uso de los soportes digitales, de allí que se tomen textos digitales que puedan ser leídos, debatidos y analizados desde una mirada más cercana a la realidad. Como plantea Dussel (2011), la presencia y uso de elementos tecnológicos en el aula redefinen este espacio. El docente al igual que el estudiante replantean sus roles y estrategias de interacción dentro del aula.

Con base en lo anterior, se presentó la secuencia didáctica crítica titulada «Los textos multimodales digitales: otras maneras de familiarizarnos con la lectura». Sus objetivos de aprendizaje fueron los siguientes: a) promover actividades de lectura multimodal digital que favorecieran el comportamiento lector; b) motivar a los estudiantes a un encuentro con la lectura de manera reflexiva y crítica 
que contribuyera en su formación personal, académica y social; c) vincular a miembros de la comunidad educativa en los espacios de vinteracción presencial y virtual que promovieran posturas críticas, así como el respeto por el otro; d) evaluar el impacto de la intervención didáctica desde la percepción de los estudiantes.

Como se observa en la Figura 1, la secuencia didáctica crítica se planteó como una estrategia dinámica, que favoreciera la interacción y participación de los estudiantes, donde relacionaran sus experiencias y saberes dando cuenta de la meta establecida, así como el trabajo en equipo, reconociendo al estudiante como un sujeto analítico, propositivo interesado por leer desde su realidad y estableciendo un comportamiento lector, que incidió, posteriormente, en sus decisiones como un sujeto social. Así mismo, al maestro como un sujeto consciente de su labor, que interactuó y ayudó en la formación de los sujetos desde una mirada orientadora y propositiva, acorde con los intereses de los estudiantes.

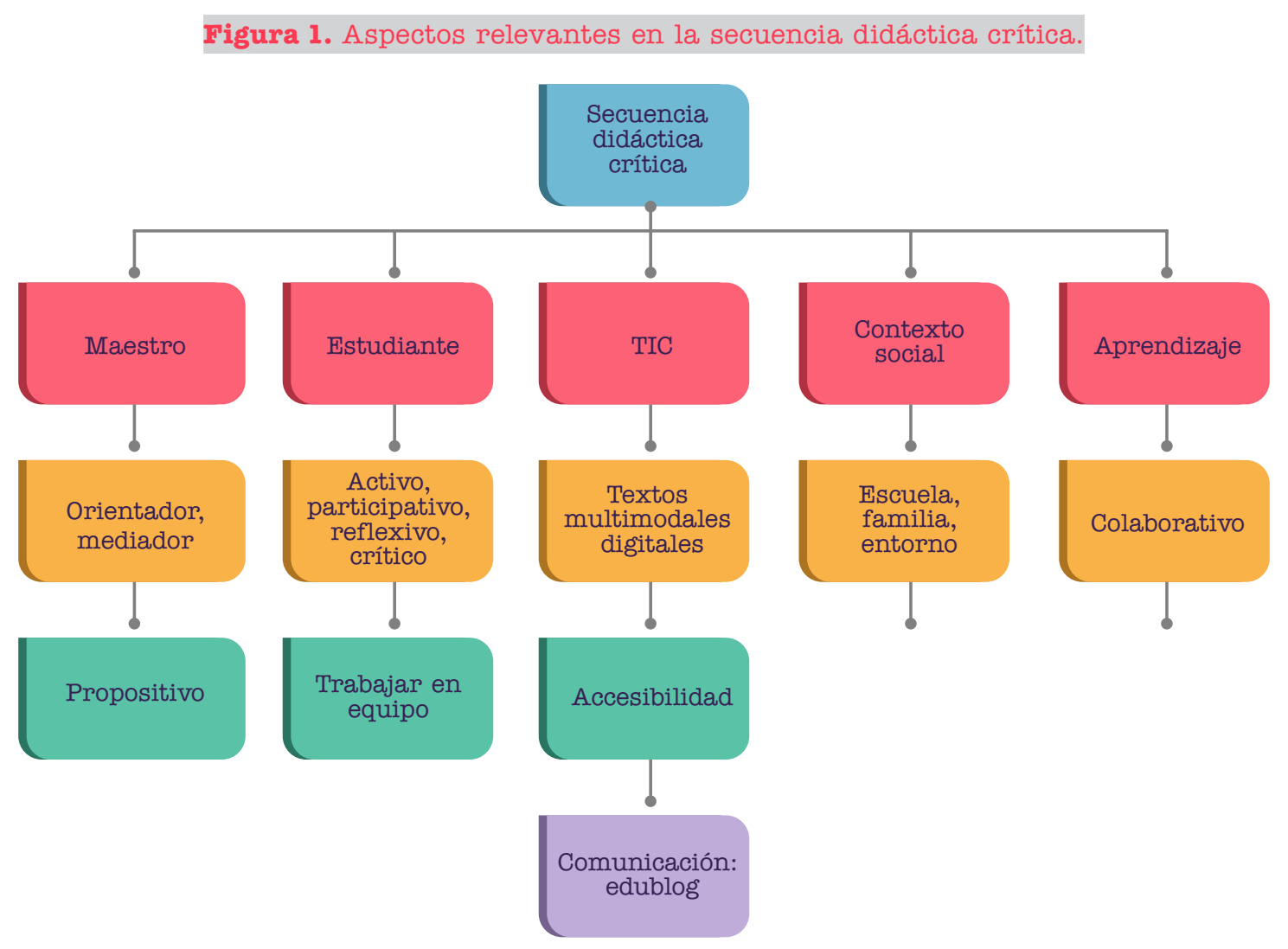

Fuente: elaboración propia. 
Para esto resultó necesario, además, establecer los momentos que orientaron la secuencia: a) actividad para activar conocimientos/ experiencias previas; b) desarrollo en donde el trabajo en equipo fue fundamental en la medida que se dio valor a sus experiencias y saberes, a la cooperación y solidaridad; c) cierre, denominado socialización de resultados, permitió evidenciar el objetivo final de las estrategias propuestas visible en las opiniones, reflexiones hechas por los estudiantes; d) evaluación formativa que dio cuenta de un proceso de retroalimentación, de autorreflexión y de experiencias que permitió analizar aquellos para mejorar o corregir en la ejecución de las estrategias.

La secuencia didáctica se conformó por tres ciclos en los cuales se abordaron textos narrativos, expositivos y argumentativos ( $F i-$ gura 2). Cada ciclo estuvo determinado por sesiones de trabajo, orientadas por actividades asociadas a los procesos de lectura: pre-lectura, lectura y post-lectura abordadas de manera transversal, teniendo presente el objeto de estudio de la investigación. Además, como mecanismo de divulgación e interacción con la comunidad educativa se creó un blog de la experiencia que se desarrollaba en el aula.

Figura 2. Diseño secuencia didáctica.

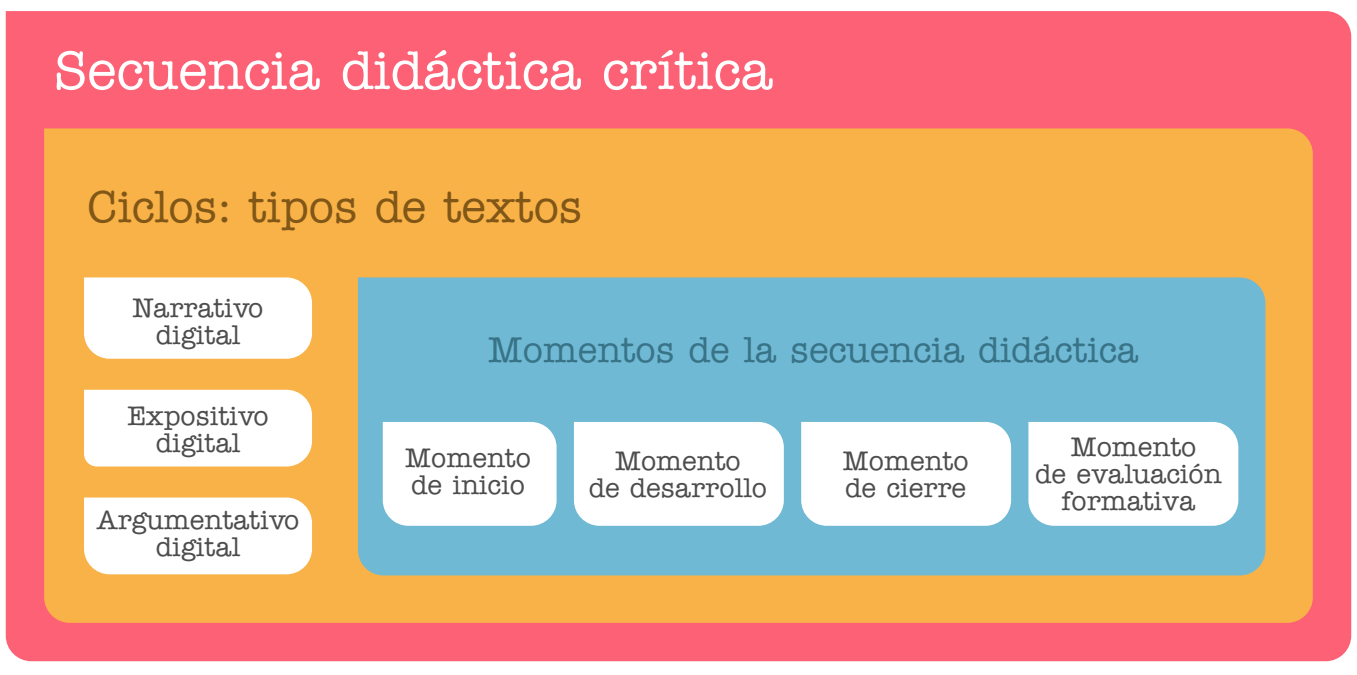

Fuente: elaboración propia. 
Al diseñar la secuencia didáctica se tuvo claridad del objetivo por seguir, de tal manera que los momentos y estrategias fueran pertinentes y eficaces. En este sentido, fue el profesor quien orientó una ruta que contribuyó a este objetivo, vinculando recursos acordes al contexto y a las necesidades, siendo consciente de que las estrategias planteadas le exigieron unas prácticas pedagógicas más rigurosas y con sentido. En consecuencia, Rivero Cárdenas, Gómez Zermaño y Abrego Tijerina (2013) señalan que «Las estrategias son competencia, en gran medida, del docente como responsable del diseño y desarrollo del proceso de enseñanza y aprendizaje» (p. 193). Esto, considerando que los recursos que actualmente se emplean exigen más compromiso del maestro en cuanto a su manejo y aceptación, de tal manera que haya un equilibrio en cuanto a lo que el estudiante espera y lo que el maestro pretende reflejar en sus prácticas.

\section{Instrumentos de investigación}

Grupo de discusión. El grupo de discusión como instrumento de recolección de información, permitió la construcción de un discurso reflexivo pensado en el reconocimiento de las fortalezas e inconvenientes que se presentaron en las categorías propuestas en la investigación, de las cuales se configuró una propuesta didáctica crítica. Para la elección del grupo de discusión se tuvo en cuenta personas con disposición de tiempo e interesadas por aportar sus saberes, necesidades e inquietudes frente al tema a plantearse. Entre ellos estuvieron: una coordinadora, una docente, dos madres de familia y un estudiante del grado seleccionado. Para su ejecución fue necesario contar previamente con un ambiente cómodo y accesible a los participantes. Como plantea Aravena et al. (2006), «La discusión será inaugurada por el investigador, quien expondrá el tema sobre el que desea que se hable» (p. 75). Se partió de una pregunta orientadora, de la cual se generaron otras a partir del discurso que se tejió entre los participantes. Finalizó en el momento que el investigador consideró que la temática planteada fue abordada satisfactoriamente.

Guía de observación. La observación como instrumento de investigación permitió al investigador ser partícipe de una serie de eventos en los cuales la población se encuentra inmersa. «Desde el paradigma cualitativo-interpretativo se considera que la penetración del investigador, en tanto más cercana a su objeto de estudio, mejores registros van a obtener; implicando observar y participar sin exclusión alguna» (Martínez, 2007, p. 75), convirtiéndose en un instrumento apropiado para conocer aspectos relevantes en la investigación desarrollada. En esta oportunidad el investigador principal se vinculó a las actividades desarrolladas por los estudiantes, siendo esta una observación participante.

Para llevar a cabo la observación se diseñó una guía, la cual se constituyó por categorías y serie de preguntas orientadoras, que determinaron la ruta de descripción y análisis de los eventos observados. De esta manera, se abordaron cinco preguntas orientadoras referidas al comportamiento lector de textos multimodales digitales y tres referidas a la didáctica crítica. Además, se tuvo en cuenta aspectos como la descripción e interpretación, de los cuales se logró proveer de información óptima a la investigación, adquiriendo mayor profundidad en el discurso (Martínez, 2007). 
Las preguntas orientadoras fueron: 1) ise evidencia en los estudiantes interés por participar en los momentos de la lectura (prelectura-lectura-lectura comprensiva)?; 2) ¿existe mayor interés de algunos tipos de textos por parte los estudiantes?; 3) ¿tienen los estudiantes la oportunidad de elegir textos para vincularlos a sus actividades académicas?; 4) ¿los estudiantes manifiestan agrado al interactuar con la lectura multimodal digital?; 5) ¿cómo es el manejo de las actividades interactivas y los soportes digitales por parte de los estudiantes?; 6) ¿el estudiante trabaja en equipo cumpliendo con los roles establecidos?; 7) ¿el estudiante toma una postura crítica ante las situaciones planteadas en las actividades?; 8) ¿se evidencia la integración del contexto a las actividades desarrolladas en clase?

La información de la guía de observación se recolectó durante el desarrollo de la secuencia didáctica crítica. Diariamente se hicieron anotaciones de lo observado en los estudiantes de tercer grado y el desarrollo de cada actividad teniendo presente el objeto de estudio a analizar. Esto permitió observar y describir aspectos y actitudes de los estudiantes, sus prácticas en el aula relevantes para la investigación. Las observaciones siempre estuvieron acompañadas de las descripciones e interpretaciones, las cuales giraron en torno a preguntas orientadoras previamente establecidas. Para la digitalización no se descartó ninguna información, considerando que todo cuanto se decía fuese válido para el análisis. En algunas observaciones se omitieron preguntas orientadoras por no tener relación alguna con la actividad por desarrollar. Para mayor organización se utilizó el programa de Microsoft Word y se procedió de manera inductiva a analizar la información con el fin de respetar los datos recolectados. Se analizaron 24 guías de observación, organizadas según el número de la pregunta abordada, de los cuales emergieron categorías y descriptores que permiten mayor interpretación de la información.

Cuestionario a estudiantes. El Cuestionario «permite recoger datos de un amplio volumen de sujetos o de una muestra para que sea representativa de la población sobre la que se pretende hacer extensivas las conclusiones obtenidas en la muestra» (Hernández, Cantín, López y Rodríguez, 2014, p. 9). De tal manera que el investigador preguntó aspectos claves de los cuales obtuvo respuesta. Seguido a esto, se agrupó, se analizó y se dieron conclusiones. De ahí, la necesidad de que las preguntas plasmadas fueran precisas, tuvo un lenguaje claro de acuerdo con la edad, y al contexto, evitando, además, la confusión en sus respuestas.

El objetivo del cuestionario fue evaluar la intervención realizada. Para tal efecto, se tuvo en cuenta sus experiencias con la lectura multimodal digital, la frecuencia de su uso, sus intereses frente a los tipos de textos, la interacción con los textos y los espacios en familia. Para ello, se plantearon 12 preguntas abiertas. El cuestionario se realizó en un solo momento al finalizar la propuesta didáctica crítica. Se pidió a los estudiantes marcar sus cuestionarios con fecha y grado correspondiente. Se explicó su objetivo, lo importante que era responder a todas las preguntas y que su desarrollo no tendría repercusiones en su rendimiento académico. Se indicó, además, como tiempo límite dos horas y que este se desarrollaría de manera individual. El método utilizado para este análisis fue el análisis de contenido, entendido como una técnica de interpretación de textos, el cual combina la observación y producción de los datos, y la interpretación o análisis de los mismos, abriendo las puertas a conocimientos de diversos aspectos y fenómenos de la vida social. Con este 
método, se pudo realizar una lectura objetiva de la información plasmada por los participantes en cada uno de los instrumentos, interpretando e infiriendo sus comentarios, dando respuesta a aspectos puntuales de la investigación. Andréu (2000) afirma que «el propósito fundamental del análisis de contenido es realizar 'inferencias' referidas fundamentalmente a la comunicación simbólica o mensaje de los datos, que tratan en general, de fenómenos distintos de aquellos que son directamente observables» (p. 3). De ahí, que se encontrara mayor sentido a la información y así alcanzar un nivel de análisis pertinente que diera respuesta a los objetivos de la investigación.

\section{Resultados}

Los resultados se presentarán acorde con las etapas de la propuesta didáctica y la relación con los instrumentos de investigación, así: a) planeación - grupo de discusión; b) implementación - guía de observación; c) evaluación - cuestionario a estudiantes.

\section{Planeación de la propuesta}

El grupo de discusión aportó a la identificación de unas categorías orientadoras para el proceso de planeación de la propuesta didáctica, buscando que esta respondiera a la realidad institucional. A continuación, se presenta una tabla (Tabla 2) que muestra las relaciones identificadas en el diálogo con los participantes entre la categoría, el descriptor y las decisiones en la planeación:

Tabla 2. Análisis grupo de discusión.

\begin{tabular}{l|l|l} 
Categoría & \multicolumn{1}{|c}{ Descriptor } & \multicolumn{1}{c}{ Decisiones } \\
\hline \multirow{3}{*}{ Hábito lector } & $\begin{array}{l}\text { Para lograrlo se requiere del } \\
\text { acompañamiento familiar y } \\
\text { escolar, motivación, conocer } \\
\text { intereses, tiempos de lectura. } \\
\text { Lectura del entorno, realidad } \\
\text { social, escolar y familiar, } \\
\text { posturas críticas, argumentos. }\end{array}$ & $\begin{array}{l}\text { Se vinculó a los padres de familia } \\
\text { en la implementación de la } \\
\text { secuencia didáctica partiendo de un } \\
\text { taller donde conocieron cómo sería } \\
\text { el trabajo con sus hijos y cómo } \\
\text { desde sus hogares, su entorno } \\
\text { social y sus saberes los podrían } \\
\text { acompañar. }\end{array}$ \\
\hline
\end{tabular}




\begin{tabular}{|c|c|c|}
\hline Categoría & Descriptor & Decisiones \\
\hline Entorno social & $\begin{array}{l}\text { Lectura del entorno. Lograr } \\
\text { niveles de comprensión, } \\
\text { interacción, análisis, } \\
\text { proposición. }\end{array}$ & $\begin{array}{l}\text { Se plantearon lecturas de textos } \\
\text { multimodales digitales relacionadas } \\
\text { con situaciones de su realidad } \\
\text { social, las cuales les generó gran } \\
\text { interés y oportunidades para } \\
\text { intervenir e interactuar en sus } \\
\text { equipos de trabajo. Así mismo, } \\
\text { proponer otros tipos de lecturas en } \\
\text { los distintos soportes. }\end{array}$ \\
\hline Rol del maestro & $\begin{array}{l}\text { El maestro es visto como } \\
\text { un sujeto tradicionalista, } \\
\text { innovador, orientador, } \\
\text { responsable e inspirador para } \\
\text { sus estudiantes. Se espera un } \\
\text { maestro innovador, orientador, } \\
\text { responsable. }\end{array}$ & $\begin{array}{l}\text { Maestro orientador, dinámico, guía, } \\
\text { atento a las inquietudes de sus } \\
\text { estudiantes. En la búsqueda de } \\
\text { sujetos autónomos, colaborativos, } \\
\text { críticos. }\end{array}$ \\
\hline Multimodal & $\begin{array}{l}\text { Uso de textos digitales, } \\
\text { videos, juegos interactivos, } \\
\text { accesibilidad, uso de las TIC. }\end{array}$ & $\begin{array}{l}\text { Implementación de textos } \\
\text { multimodales digitales sujetos a } \\
\text { situaciones del entorno e interés } \\
\text { del estudiante que contribuyen a } \\
\text { reconocer aspectos que inciden } \\
\text { en su comportamiento lector. } \\
\text { Desarrollo de habilidades técnicas } \\
\text { en cuanto al uso de las TIC. }\end{array}$ \\
\hline $\begin{array}{l}\text { Trabajo en } \\
\text { equipo }\end{array}$ & $\begin{array}{l}\text { Promover el liderazgo, respeto, } \\
\text { solidaridad, diálogo, libertad } \\
\text { de opiniones. }\end{array}$ & $\begin{array}{l}\text { Consolidación de equipos de trabajo } \\
\text { que promuevan el aprendizaje } \\
\text { colaborativo, el liderazgo, la } \\
\text { escucha, la autonomía, opiniones y } \\
\text { posturas críticas frente a los textos } \\
\text { presentados, los cuales guardan } \\
\text { relación con su entorno social. }\end{array}$ \\
\hline
\end{tabular}

Fuente: elaboración propia.

Este diagnóstico conllevó a tomar decisiones para el diseño de la propuesta didáctica crítica en donde fuese visible el entorno como aspecto integrador en las lecturas planteadas. Además, surgió la necesidad de diseñar una sala de informática, donde el estudiante pudiera interactuar con dichas herramientas de manera significativa en lo que concierne a aspectos académicos tratados a partir de la propuesta didáctica. De tal manera que él hiciera lecturas de textos multimodales digitales, trabajara en equipo, interactuara con sus semejantes y así vincular a la comunidad educativa en cada una de las actividades propuestas, siendo esto una de las sugerencias propuestas por los participantes del grupo de discusión. Así mismo, se propuso la lectura de diversos tipos de textos multimodales digitales: narrativos, expositivos y argumentativos que conlleven a conocer la relación con los textos, la frecuencia con la que los leen, sus intereses lectores, entre otros aspectos. 


\section{Implementación}

\section{de la propuesta}

Seleccionado el grupo de estudiantes con quien se iba a implementar la propuesta didáctica, se realizó una reunión con la comunidad educativa, padres, docentes y directivos docentes para socializar cada uno de los aspectos de la investigación y cuál sería su participación en este proceso. Se brindó un espacio para aclarar dudas y sugerencias. Inicialmente, se desarrolló un taller con los padres de familia del grado tercero en el cual se les orientó sobre el trabajo por ejecutar con sus hijos y cómo desde sus hogares ellos contribuirían a fomentar el comportamiento lector a través de la interacción con lecturas multimodales digitales, que incentivaran a su vez la socialización de experiencias. De igual manera, se buscó vincularlos en espacios de interacción presencial y virtual que promovieran posturas críticas, así como el respeto por el otro. Así se dio la oportunidad de ser partícipes del edublog en el cual se visualizó cada una de las actividades desarrolladas y publicaciones de los estudiantes, y de ellos mismos. Dicho blog quedó abierto para futuras publicaciones y actividades.

Durante el desarrollo de la secuencia didáctica se buscó, a partir del uso de textos multimodales digitales, su incidencia en el comportamiento lector de los estudiantes. Para ello, se propusieron temáticas que guardaran relación con su entorno social. Aspectos como la convivencia, los miedos, el cuidado de la vida, el cuidado del medio ambiente, la alimentación, el bullying y la familia resultaron ser interesantes tanto para hacer lecturas desde sus realidades a partir de los textos multimodales digitales como para establecer diálogos y posturas críticas.
Se estableció la transversalidad de temáticas en áreas como: Lengua Castellana, Ciencias Sociales, Tecnología e Informática y, Ética y Valores pretendiendo con ello dar significado a los aprendizajes de los estudiantes, reconociendo que es posible establecer relaciones entre los saberes adquiridos y construidos a partir de la interacción social. En este punto, los planes de áreas fueron flexibles ante sus necesidades e intereses con relación al contexto social. Por esta razón, más allá de brindar conceptos aislados, estos se fundamentan bajo aprendizajes contextuados, alrededor del trabajo en equipo.

Un aspecto que se observó en las primeras sesiones fue el temor de cuatro estudiantes por enfrentarse al uso del computador. Indicaron que nunca habían tenido acceso a esta herramienta en las instituciones educativas donde estudiaron anteriormente y tampoco contaban con esta en sus hogares. Otros estudiantes narraron que sus padres no les dejaban utilizar libremente la tableta, el computador o el celular, lo cual les impedía interactuar con lecturas y otras actividades digitales. Mientras que otros mencionaban tener conocimiento y habilidades para utilizarlas y se ofrecieron a ayudar a sus compañeros en cuanto al manejo técnico.

Inicialmente, los estudiantes preferían que quienes ya habían interactuado con herramientas tecnológicas fueran quienes las manipularan y ellos se limitaban a ver. Indicaban que en el hogar solo lo usaban sus padres o hermanos mayores, otros lo utilizaban solo para juegos. Sin embargo, con el trabajo en equipo se evidenció en el transcurso de las actividades, la colaboración para con estos estudiantes, al punto que al finalizar la secuencia didáctica estos estudiantes se mostraron activos, con autonomía y seguridad para realizar las actividades y buscar otros espacios para utilizar las herramientas tecnológicas. 
Se evidenció en el desarrollo de las sesiones que quienes iban adquiriendo mayor habilidad técnica en el uso de los soportes tecnológicos realizaban las actividades por sí solos con mayor seguridad. Es probable que el acompañamiento, la disposición de tiempo y una sala de informática alterna hayan generado espacios significativos para el manejo de las herramientas digitales, así como de las actividades propuestas. Todo lo anterior incidió en que los estudiantes se motivaran al encuentro con situaciones o experiencias diversas a partir de las lecturas contextuadas. Así como interés por repetir las actividades una y otra vez experimentando en ellas sensaciones o emociones diversas.

Ciclo 1: textos narrativos digitales. Para el primer ciclo se planteó el uso de textos narrativos digitales, con el propósito de favorecer espacios de lectura con textos cercanos a los estudiantes que conllevaron a la reflexión y cuidado de su ser, construcción de pautas de convivencia y conciencia en cuanto al respeto de normas dentro y fuera de sus casas. Este estuvo estructurado en tres sesiones relacionadas con las pautas de convivencia, a la obediencia y a ser sujetos seguros de sí mismos. Los estudiantes escogieron en su mayoría escuchar los audios porque les daba la oportunidad de leer las imágenes, discriminar colores, movimientos y compararlas con las lecturas de otros cuentos. Se mostraron emocionados al conocer otras maneras de leer, pues con frecuencia en la escuela los textos suelen ser impresos. Se socializaron sus experiencias con los textos y sus posturas frente a los conceptos abordados.

Al iniciar las sesiones de trabajo, la mayoría de los estudiantes tenían preferencias por los textos analógicos, esto por ser los más próximos para sus lecturas en casa y en espacios como la biblioteca municipal o encuentros literarios que hacian en sus barrios. Sin embargo, en las sesiones siguientes, los estudiantes expresaron darles otros usos a sus herramientas tecnológicas, buscando y leyendo textos multimodales digitales. Solicitaban, además, títulos o páginas que les pudieran servir. De tal manera que los textos digitales fueron tomando fuerza entre sus intereses. Para CERLALC (2015) «Leer y dialogar crean lazos de identidad y corresponsabilidad entre los sujetos. Esta posibilidad de intercambiar experiencias ha superado los escenarios físicos y los ha transformado en universales» (p. 25). De allí que la imagen, el sonido, el movimiento en textos como videos, historietas, afiches, propagandas permitió en el transcurso de las sesiones que los estudiantes hicieran sus propias lecturas, generando una construcción de significados a partir de sus saberes previos, experiencias y conceptos abordados en el aula, los cuales permitieron establecer espacios de diálogo en torno a posiciones personales, a partir de sus realidades sociales.

Ciclo 2: textos expositivos digitales. Para el segundo ciclo se planteó el uso de textos expositivos digitales con el propósito de familiarizar al estudiante con este tipo de textos y a su vez comprender situaciones de su realidad social que le permitan reflexionar y contribuir al cuidado de su entorno. Este estuvo estructurado en tres sesiones relacionadas con el cuidado del medio ambiente y su entorno cercano. Los estudiantes se sintieron motivados por leer textos a partir de la imagen y el color, lo cual se reflejó en sus búsquedas alusivas a la temática.

Este ciclo da cuenta de la relación existente entre la lectura y el contexto social, donde el estudiante lee, se divierte, se informa, opina y construye saberes a partir de su entorno. Se convierte la lectura en un espacio ameno de 
interacción que favorece el aprendizaje colaborativo, así como la vinculación de la familia en experiencias significativas. En este punto de la propuesta, los estudiantes no han considerado la lectura de los textos propuestos como una imposición o de carácter académico; por el contrario, demuestran gusto. Tanto así que en compañía de sus padres han realizado y socializado sus búsquedas entre las cuales se encuentran videos, afiches y noticias.

Los estudiantes sintieron mayor interés por los afiches por encontrar, según ellos, «una manera más bonita de comunicarse con los compañeros sobre temas que a veces son difíciles de explicar». Esto se debe quizás a que los textos expositivos han sido poco utilizados en actividades académicas, o que, a pesar de estar en contacto en otros contextos, no conocían su nombre técnico, lo cual ha motivado a conocer más de ellos, cómo se construyen y cuál es su función. Es entonces, a partir de la curiosidad de los estudiantes, en el momento en que las estrategias docentes deben emerger e invitarlos a participar de los diversos textos que se ofrecen en los distintos soportes, de la tal manera que sean ellos quienes, a partir de su interacción, decidan realmente cuáles son sus verdaderos intereses.

Ciclo 3: textos argumentativos digitales. El objetivo de este ciclo fue establecer la interacción de los estudiantes con los textos argumentativos digitales e identificar su estructura, permitiendo a su vez expresar sus posturas frente a determinados temas que incidieran en su vida o en la de sus semejantes. Posteriormente, era necesario construir argumentos que defendieran las posturas alrededor de dichos textos, donde se abordaran temáticas de interés para los estudiantes.
El ciclo se constituyó en tres sesiones en las que se abordaban temáticas sobre la alimentación y la salud, la familia y el bullying. La lectura en equipo permitió conocer las estrategias que los estudiantes utilizaron para su comprensión e interpretación, develando sus posturas en torno a los textos y sus diversos recursos semióticos. Así mismo, que los estudiantes por turnos discutieran, analizaran y propusieran argumentos, estableciendo una relación con la realidad que viven. En sus búsquedas los estudiantes prefirieron los videos, historietas, infografías y afiches que hablaban del tema y en menor cantidad los textos escritos.

Al culminar el tercer ciclo se notó en los estudiantes interés por la realización de búsquedas, de tal manera que pudieran argumentar sus posturas ante sus compañeros. Se abordaron temáticas conocidas por ellos, las cuales permitieron que se establecieran espacios de lectura y discusión acordes con sus experiencias. De allí que se conocieran algunos comentarios de los estudiantes sobre temas como: la sana alimentación y el ejercicio, el bullying y la familia. De igual manera, los estudiantes junto a sus padres propusieron lecturas que ayudaron a documentar sobre las temáticas propuestas. Esto generó en los estudiantes mayor conciencia y respeto por sus compañeros, su vida y su familia.

Al finalizar el desarrollo de la secuencia didáctica aumentó notablemente la lectura de textos multimodales digitales al punto que los estudiantes solicitaron espacios en otras áreas para que se ejecutara este tipo de lecturas. Para los estudiantes la lectura multimodal digital promueve espacios y encuentros lectores motivantes, tanto a nivel cognitivo como social, que a su vez contribuye a tener otros espacios de diálogo e interacción como los propuestos en 
cada una de las sesiones desarrolladas. Los estudiantes demostraron interés en este tipo de actividades porque para ellos es más motivante que transcribir constantemente un contenido, escuchar a la maestra o realizar fichas de actividades. Además, manifestaron agrado por leer y escribir «de otra manera», de comunicar sus mensajes u opiniones de forma divertida y $\sin$ temor a la burla.

Cinco estudiantes se sintieron desmotivados porque no cuentan con herramientas digitales en sus hogares para continuar con sus actividades, no cuentan con el apoyo de sus padres para su uso, no tienen permiso para visitar la biblioteca o asistir a otros espacios del municipio. Otros estudiantes indicaron sentirse motivados porque compartieron con sus padres lo realizado en su aula de clase lo cual ayudó a establecer espacios de lectura en sus artefactos tecnológico. Sus padres los acompañan en sus lecturas, a algunos les están dando mayor tiempo de uso, otros estudiantes indican que ya se interesan por leer noticias y leen en casa afiches. Así mismo, algunos padres indicaron que sus hijos estaban dando un uso más significativo a las herramientas digitales en sus hogares.

\section{Evaluación de la propuesta}

Al indagar con los estudiantes cuáles fueron las lecturas multimodales digitales que más les gustaron, de manera igualitaria se refirieron a los textos narrativos y argumentativos digitales, indicando principalmente que les generó diversión y goce. Posteriormente, indicaron que estos tipos de lecturas les permitieron aprender y reflexionar sobre aspectos relacionados con el respeto, al cuidado de su entorno, a la convivencia escolar.
Con respecto a los textos narrativos digitales un estudiante indicó su gusto al expresar: «porque aprendí a cuidarme» ratificando que, así como existe diversión al leerse, también emergen sentimientos y patrones que en este caso son de autocuidado, donde el entorno social se evidencia y los textos que lee inciden en su comportamiento y en sus decisiones. Encontrando, quizás, relaciones entre los textos multimodales leídos y los patrones de crianza propios de su hogar. Como plantea Cordón (2010) el uso de soportes digitales favorece el comportamiento lector de los estudiantes al establecerse espacios de lectura motivantes que vinculan el ámbito social del estudiante.

Resulta ser importante para los estudiantes leer en soportes digitales porque les permite interactuar con diversidad de textos y pueden hacerlo en cualquier momento y lugar. Interactuar con lo digital se convierte para el estudiante en una herramienta facilitadora de información e interpretación, que a su vez fomenta espacios de interacción, ya sea de manera personal o virtual. Así mismo, fomenta aquellas prácticas letradas vernáculas (Cassany, 2009) que permiten a su vez reconocer aspectos de su comportamiento lector. El interés de los estudiantes radicó, además, en el interés por los recursos semióticos encontrados en cada uno de los textos como la imagen, el color, el movimiento, donde el estudiante hacía sus lecturas y las relacionaba con sucesos de su entorno.

Teniendo en cuenta cada una de las actividades de lectura multimodal digital realizadas por los estudiantes, ellos consideran que su forma de leer y comprender ha mejorado. Expresan que leen diversos tipos de textos con más facilidad, con menos frecuencia solicitan ayuda cuando no comprenden, tienen mayor atención, opinan 
y plantean propuestas de aquello que leen. Por lo anteriormente dicho, los estudiantes se sienten más seguros de sus habilidades y saberes adquiridos. Como lo plantean Alpuche Hernández y Vega Pérez (2014), la incorporación de acciones y estrategias en sus lecturas permiten favorecer el comportamiento lector en los estudiantes. De allí la propiedad con que ingresaron a desarrollar cada una de las actividades propuestas en el edublog, así como las búsquedas y comentarios propuestos. Es así, que indican en sus preferencias las búsquedas de textos narrativos digitales.

Sus visitas al edublog para realizar las actividades fueron casi diarias, contando las que se hacían en horarios de clase. Algunos estudiantes comentaron haber ingresado los fines de semana y en horario contrario a la jornada escolar. Sin embargo, la mayoría de los estudiantes expresaron haber ingresado al edublog solo en la institución porque en su casa no había conexión a internet o sus padres no les prestaban las herramientas digitales. Si bien en los hogares no hay herramientas tecnológicas, los adultos tampoco se interesan por que ellos o sus hijos utilicen las que están a su servicio en establecimientos públicos, como la biblioteca municipal. Esto se puede explicar por las relaciones de las familias en la cultura digital, dadas por los prejuicios que se le han dado a las TIC, a la negación de hacer parte de la era digital o por sentirse excluidos al no poder fortalecer sus habilidades tecnológicas.

Los estudiantes expresaron sentirse felices y cómodos utilizando el computador para leer porque tuvieron mayor acceso al recurso tecnológico, hicieron búsquedas de textos y antes no le habían dado este uso. A esto se suma que las lecturas tenían una estructura diferente a la que diariamente se les presenta como son el uso de herramientas tecnológicas, el uso de distintos recursos semióticos, la lectura no lineal, la búsqueda de textos en línea. El uso de diversos recursos semióticos generó mayor interés en los estudiantes. «La lectura ya no puede reducirse a la decodificación del sistema alfabético, tanto porque no basta con descifrar para leer, como por el hecho de que el código alfabético no es el único sistema de signos que es susceptible de ser leído» (Gutiérrez, 2008, p. 146). Es por ello, que en los espacios de diálogo los estudiantes reafirmaban su interés por participar de las actividades propuestas, ya que no lo veían como un compromiso escolar.

Afirmaron que el acompañamiento de la docente fue importante, en la medida en que se convirtió en una orientadora de sus procesos, quien los escuchaba y atendía cuando lo requerían, eran ellos quienes desarrollaban en sus equipos de trabajo sus actividades. La docente se convirtió en una mediadora entre el estudiante y la lectura, de alguna manera determinó el encuentro del estudiante con los diversos tipos de textos. Se involucró en la lectura orientando cada uno de los momentos de lectura: prelectura, lectura y poslectura e incentivando la búsqueda de nuevos textos que determinaran sus intereses. Estableció espacios de reflexión que evidenciaron aspectos determinantes en cuanto a su comportamiento lector. Se involucró en los procesos de aprendizaje, favoreciendo en los estudiantes la toma de decisiones, la interpretación, sus posturas frente a situaciones que emergen de su contexto social, la autorreflexión, así como la aplicación de valores que fortalecieron su personalidad. 


\section{Conclusiones}

Los miembros de la comunidad educativa demostraron un notable interés en que sus hijos y estudiantes fomenten o fortalezcan su comportamiento lector. Sin embargo, las herramientas con que cuentan en sus hogares e instituciones (escuela, biblioteca, parque educativo) están siendo subutilizadas y el tiempo que dedican a la lectura, padres e hijos, es escaso. Por esto, consideran que el apoyo y acompañamiento debe ser dado desde la escuela, la cual cuenta con los recursos tecnológicos y personal idóneo e innovador, para motivar a los estudiantes a la lectura. Ante esta postura y la de autores como Yubero Jiménez y Larrañaga Rubio (2010), favorecer el comportamiento lector es un trabajo compartido entre la escuela y la familia y es en este acompañamiento que es posible ver los resultados que se esperan. Por ello, se piensa en una propuesta didáctica donde esté de la mano el apoyo de los padres quienes velarán también porque sus hijos se sientan motivados por la lectura y encuentren en ella una relación con su realidad social, la cual contribuye a formarse como un sujeto íntegro consciente de su rol en el entorno donde vive.

Reconociendo sus intereses por leer, por descubrir textos de su agrado de acuerdo con su edad y entorno, se buscó plantear una propuesta didáctica crítica que, si bien incidió en el comportamiento lector, también permitió mejorar formas de pensamiento, reconocer y fomentar situaciones que afectaron de manera positiva su vida, donde se cuestionó, indagó y logró motivar a quienes estaban a su alrededor a vincularse a la lectura. Se buscó, además, hacer evidente las reflexiones realizadas a partir de los textos multimodales digitales empleados, en los que el estudiante como miembro de un entorno determinado opinó, analizó, tomó posturas que repercutieron en su quehacer personal, escolar y social.

Al iniciar la secuencia didáctica crítica y a pesar de haberse establecido pautas de convivencia, costó un poco concienciar a los estudiantes sobre el respeto por el otro, a sus opiniones y posturas. Eran pocos los estudiantes que se atrevían a dar sus puntos de vista con propiedad y a cuestionar las decisiones de otros compañeros. En el transcurso de las sesiones los estudiantes notaron que lo que se comentaba tenía sentido con su entorno y empezaron libremente a aportar opiniones, a justificarlas y a proponer ideas en pro del bienestar de otros. Algunos estudiantes se preparaban antes de hablar queriendo siempre ser claros en sus opiniones y comentarios. Mejoraron poco a poco el análisis y asociación entre las situaciones y lecturas planteadas con su realidad.

Implementar en la secuencia didáctica, el uso de los textos expositivos fomentó el interés por estos tipos de textos, elaborar hipótesis e inferencias que permitieron la construcción de nuevos significados a partir de sus pre saberes o predicciones, realizar búsquedas digitales, y diversas maneras de comunicar una idea o información. Permitió, además, que los estudiantes establecieran relaciones entre el texto y situaciones de su entorno. Estas relaciones convocaron a que los estudiantes tomaran conciencia de la importancia del agua, en tanto contribuyó a reflexionar sobre los cuidados que se le deben dar a nivel personal y social. Que los estudiantes retomaran información del texto base para sus nuevas búsquedas fue una oportunidad para interactuar e interesarse por otros tipos de textos. 
Con el paso de los días se evidenció en los estudiantes mayor atención e interés por participar en las lecturas propuestas en la medida que comprendieron que este no fue solo un trabajo de clase, sino un espacio que permitió la reflexión, la interacción, la escucha, el diálogo y el acceso a miembros de su familia; a tal punto que algunos padres hicieron comentarios de los textos multimodales planteados en las sesiones de trabajo. Participaciones como las anteriores generaron en los estudiantes mayor interés por leer. En los espacios de discusión y trabajo en equipo lograron exponer sus posturas con mayor confianza, de tal manera que propusieron textos multimodales digitales a sus compañeros de forma verbal en las sesiones y a través del edublog. Algunos de estos textos se leyeron en el aula y otros en los espacios que tenían los estudiantes en el hogar para dicho fin.

Como se evidencia en las sugerencias de lectura anteriores, a pesar de presentarse a los estudiantes a lo largo del desarrollo de la propuesta didáctica crítica textos multimodales narrativos, expositivos y argumentativos digitales, sus intereses y puntualmente sus búsquedas continúan siendo los textos de corte narrativo. Camps (2002) plantea que los materiales de lectura a los que acceden los individuos están ligados a los procesos de lectura y escritura de los círculos en los que conviven. Una de las posibles causas por la que los estudiantes retoman los textos narrativos, a pesar de que les presentan otros, tiene que ver con que en su casa o en los escenarios culturales en los que ellos se desarrollan sean los textos narrativos los que mayor oferta tengan para que ellos lean, escriban y alcancen los niveles de comprensión lectora.
Para finalizar, los estudiantes comentaron sentirse motivados con las actividades propuestas en cada una de las sesiones. Indicaron haberles gustado las lecturas multimodales digitales, participar con sus trabajos y comentarios en el blog, trabajar en equipo y el rol de la docente. Lo anterior da pie para que propuestas como la diseñada contribuyan de manera significativa en la formación integral de los estudiantes. Sus expresiones en cuanto a la lectura permiten pensar que la investigación tuvo incidencia en su comportamiento lector, en la medida que sus intereses cambiaron, los espacios de diálogo frente a los textos son más significativos y motivan a otros compañeros a participar de espacios de lectura.

\section{Referencias}

Alpuche Hernández, A., y Vega Pérez, L. O. (2014). Predicción del comportamiento lector a partir de la autoeficacia. Revista mexicana de investigación educativa, 19(60), 241-266. Recuperado de https://bit.ly/2AoCDZc

Andréu, J. (2000). Las técnicas de Análisis de Contenido: una revisión actualizada. Fundación Centro Estudios Andaluces, Universidad de Granada, 10(2), 1-34. Recuperado de http://public.centrodeestudiosandaluces.es/pdfs/S200103.pdf

Aravena, M., Kimelman, E., Micheli, B., Torrealba, R., \& Zúñiga, J. (2006). Investigación educativa I. Recuperado de https://bit.ly/2TOmaGB

Camps, A. (2002). Hablar en clase, aprender lengua. Aula de Innovación Educativa (111), 6-10. Recuperado de https://www.academia.edu/25950188/ Hablar_en_clase_aprender_lengua 
Cassany, D. (2009). Para ser letrados. Voces y miradas sobre la lectura. Barcelona: Paidós Ibérica. Recuperado de https://bit.ly/2TOWOIK

CERLALC. (2015). Alfabetización: una ruta de aprendizaje multimodal para toda la vida. Consideraciones sobre las prácticas de lectura y escritura para el ejercicio ciudadano en un contexto global e intercomunicado. Recuperado de https://bit.ly/2TVKQ02

Cordón, J. (2010). De la lectura ensimismada a la lectura colaborativa: nuevas topologías de la lectura en el entorno digital. Salamanca: Ediciones Universidad de Salamanca. Recuperado de http://eprints.rclis.org/15441/1/ lecturaensimismada.pdf

Cuesta, R., Mainer, J., Mateos, J., Merchán, J., y Vicente, M. (2005). Didáctica crítica. Allí donde se encuentran la necesidad y el deseo. Revista ConCiencia Social (9), 17-54. Recuperado de https://bit.ly/2E2ochV

Doria, R., \& Castro, M. (2012). La investigación-acción en la comprensión de las prácticas de enseñanza de los maestros de lenguaje. Lenguaje, 40(2), 447468. Recuperado de http://www.scielo.org.co/ pdf/leng/v40n2/v40n2a07.pdf

Dussel, I. (2011). Aprender y enseñar en la era digital ¿Vino Viejo en odres nuevos? Debates sobre los cambios en las formas de enseñar y aprender con nuevas tecnologías. Memorias VI Foro latinoamericano de Educación; Educación y nuevas tecnologías: los desafíos pedagógicos ante el mundo digital, 1-32. Recuperado de https://bit.ly/2P510jK

Fernández-Díaz, E., \& Calvo, A. (2013). Estrategias para la mejora de la práctica docente. Una investigación acción colaborativa para el uso innovador de las TIC. Revista Electrónica Interuniversitaria de Formación del Profesorado, 16(2), 121-133. Obtenido de http://www.redalyc. org/articulo.oa?id=217029557009
Gértrudix Barrio, F., y Ballesteros Ávila, V. (2014). El uso de herramientas 2.0 como recursos innovadores en el aprendizaje de niños y niñas en Educación Infantil. Un estudio de caso de investigación-acción. Edutec. Revista Electrónica de Tecnología Educativa, 0(48), a274. doi: https:// doi.org/10.21556/edutec.2014.48.60

Gimeno. (1995). Teoría crítica de la educación. una alternativa educativa para una sociedad en crisis. Madrid: UNED.

Gutiérrez. (2008). Leer digital: la lectura en el entorno de las nuevas tecnologías de la información y la comunicación. Signo y Pensamiento, 28(54) 144-163. Recuperado de http://www.redalyc. org/articulo.oa?id=86011409010

Hernández, R., Fernández, C., y Baptista, P. (2010). Metodología de la investigación. México: McGrawHill, $5 a$ ed. Recuperado de https://www.esup.edu. pe/descargas/dep_investigacion/Metodologia\%20 de\%20la\%20investigaci\%C3\%B3n\%205ta\%20 Edici\%C3\%B3n.pdf

Hernández., A., Cantin., S., López, N., y Rodríguez, M. (2014). Estudio de encuestas. Recuperado de https://bit.ly/1Cc1b4o

Klafki, W. (1990). Sobre la relación entre didáctica y metódica. Revista Educación y Pedagogía, 2(5), 85-108. Recuperado de https://bit.ly/2QkJ89y

Martínez, L. (2007). La observación y el diario de campo en la definición de un tema de investigación. Revista Perfiles Libertadores (4), 73-80.

Pérez Abril y Rincón Bonilla. (2009). Actividad, Secuencia Didáctica y Pedagogía por Proyectos: Tres alternativas para la organización del trabajo didáctico en el campo del lenguaje. Bogotá: CERLALC. Recuperado de https://bit.ly/2PVi8Ot

Rivero Cárdenas, I., Gómez Zermeño, M. G., y Abrego Tijerina, R. F. (2013). Tecnologías educativas y estrategias didácticas: criterios de selección. Revista Educación y Tecnología, (3), 190-206. Recuperado de https://dialnet.unirioja.es/servlet/ articulo?codigo $=4620616$ 
Rodríguez, M. (1997). Hacia una didáctica crítica. Madrid: La Muralla.

Yubero Jiménez,S.,\&LarragañaRubio,E. (2010). Elvalor de la lectura en relación con el comportamiento lector. Un estudio sobre los hábitos lectores y el estilo de vida en niños. Ocnos: Revista de estudios sobre lectura, (6), 7-20. Recuperado de https://bit. ly/2PXd2RK 